\title{
QUEEN'S
UNIVERSITY
BELFAST
}

\section{A new energy spectrum reconstruction method for time-of-flight diagnostics of high-energy laser-driven protons}

Milluzzo, G., Scuderi, V., Alejo, A., Amico, A. G., Booth, N., Borghesi, M., Cirrone, G. A. P., Cuttone, G., Doria, D., Green, J., Kar, S., Korn, G., Larosa, G., Leanza, R., Margarone, D., Martin, P., McKenna, P., Petringa, G., Pipek, J., ... Schillaci, F. (2019). A new energy spectrum reconstruction method for time-of-flight diagnostics of high-energy laser-driven protons. Review of Scientific Instruments, 90(8), [083303].

https://doi.org/10.1063/1.5082746

Published in:

Review of Scientific Instruments

Document Version:

Publisher's PDF, also known as Version of record

Queen's University Belfast - Research Portal:

Link to publication record in Queen's University Belfast Research Portal

Publisher rights

(C) 2019 The Authors. This work is made available online in accordance with the publisher's policies. Please refer to any applicable terms of use of the publisher.

\section{General rights}

Copyright for the publications made accessible via the Queen's University Belfast Research Portal is retained by the author(s) and / or other copyright owners and it is a condition of accessing these publications that users recognise and abide by the legal requirements associated with these rights.

Take down policy

The Research Portal is Queen's institutional repository that provides access to Queen's research output. Every effort has been made to ensure that content in the Research Portal does not infringe any person's rights, or applicable UK laws. If you discover content in the Research Portal that you believe breaches copyright or violates any law, please contact openaccess@qub.ac.uk. 


\section{A new energy spectrum reconstruction method for time-of-flight diagnostics of high-energy laser-driven protons}

Cite as: Rev. Sci. Instrum. 90, 083303 (2019); https://doi.org/10.1063/1.5082746

Submitted: 23 November 2018 . Accepted: 16 July 2019. Published Online: 12 August 2019

G. Milluzzo, V. Scuderi, A. Alejo (D, A. G. Amico (D), N. Booth, M. Borghesi, G. A. P. Cirrone, G. Cuttone,

D. Doria (D), J. Green, S. Kar, G. Korn, G. Larosa (D), R. Leanza, D. Margarone, P. Martin, P. McKenna (D),

C. Petringa (D), J. Pipek, L. Romagnani, F. Romano, A. Russo, and F. Schillaci

\section{ARTICLES YOU MAY BE INTERESTED IN}

Characterizing atomic magnetic gradiometers for fetal magnetocardiography

Review of Scientific Instruments 90, 085003 (2019); https://doi.org/10.1063/1.5091007

Revised error calibration model of linear accelerometer on precision centrifuge

Review of Scientific Instruments 90, 085002 (2019); https://doi.org/10.1063/1.5089603

Studies of multilayer composite materials obtained by laser printing

AIP Conference Proceedings 2140, 020037 (2019); https://doi.org/10.1063/1.5121962

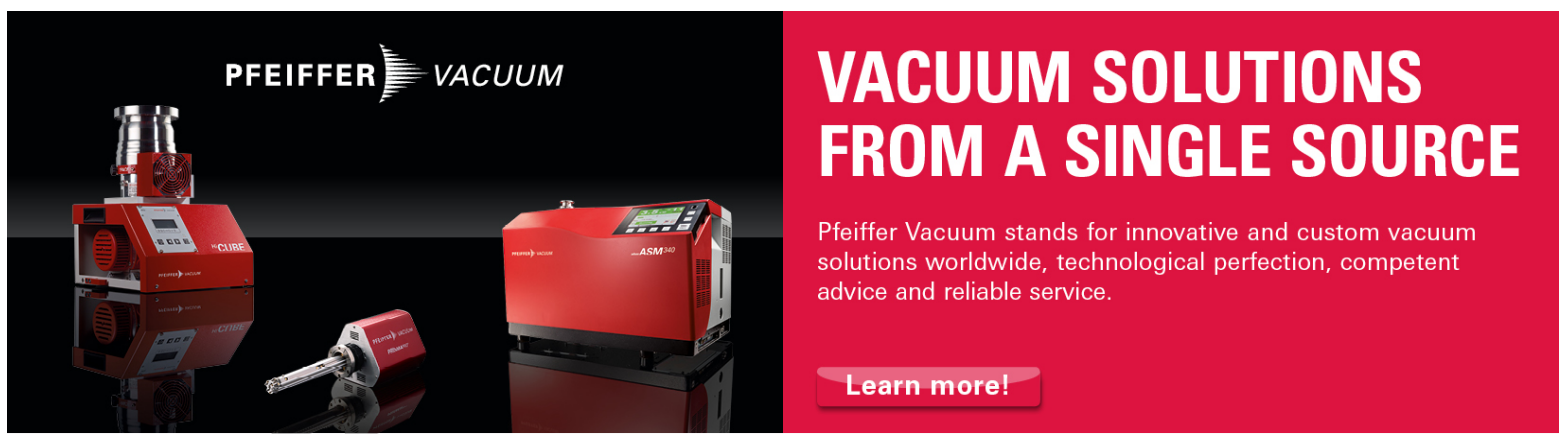




\title{
A new energy spectrum reconstruction method for time-of-flight diagnostics of high-energy laser-driven protons
}

Cite as: Rev. Sci. Instrum. 90, 083303 (2019); doi: 10.1063/1.5082746

Submitted: 23 November 2018 - Accepted: 16 July 2019 •

Published Online: 12 August 2019

\author{
G. Milluzzo, ${ }^{1,2,3, a)}$ V. Scuderi, ${ }^{2,4}$ A. Alejo, ${ }^{1}$ (D) A. G. Amico, ${ }^{2}$ (D) N. Booth, ${ }^{5}$ M. Borghesi, ${ }^{1}$ G. A. P. Cirrone, ${ }^{2}$ \\ G. Cuttone, ${ }^{2}$ D. Doria, ${ }^{1,6}$ (D) J. Green, ${ }^{5}$ S. Kar, ${ }^{1}$ G. Korn, ${ }^{4}$ G. Larosa, ${ }^{2}$ (D) R. Leanza, ${ }^{2}$ D. Margarone, ${ }^{4}$ P. Martin, \\ P. McKenna, ${ }^{7}$ (D) Cetringa, ${ }^{2}$ (D) J. Pipek, ${ }^{2}$ L. Romagnani, ${ }^{1,8}$ F. Romano, ${ }^{2,9}$ A. Russo, ${ }^{2}$ and F. Schillaci ${ }^{3,}$
}

\begin{abstract}
AFFILIATIONS
${ }^{1}$ Centre for Plasma Physics, School of Mathematics and Physics, Queen's University Belfast, Belfast BT7 1NN, United Kingdom ${ }^{2}$ INFN-Laboratori Nazionali del Sud (LNS-INFN), Via S Sofia 62, Catania 95123, Italy

${ }^{3}$ Physics and Astronomy Department, University of Catania, Via S Sofia 64, Catania 95123, Italy

${ }^{4}$ Institute of Physics ASCR, v.v.i. (FZU), ELI-Beamlines Project, 18221 Prague, Czech Republic

${ }^{5}$ Central Laser Facility, STFC Rutherford Appleton Laboratory, Didcot OX11 OQX, United Kingdom

${ }^{6}$ Extreme Light Infrastructure-Nuclear Physics (ELI-NP), Horia Hulubei Institute for Nuclear Physics (IFIN-HH), Str. Reactorului no. 30, P.O. Box MG-6, Măgurele-Bucharest 077126, Romania

${ }^{7}$ Department of Physics, SUPA, University of Strathclyde, Glasgow G4 ONG, United Kingdom

${ }^{8}$ LULI, Ecole Polytechnique, CNRS, CEA, UPMC, 91128 Palaiseau, France

${ }^{9}$ National Physical Laboratory, Hampton Rd., Teddington TW11 OLW, Middlesex, United Kingdom
\end{abstract}

a) Electronic mail: g.milluzzo@qub.ac.uk

\begin{abstract}
The Time-of-Flight (TOF) technique coupled with semiconductorlike detectors, as silicon carbide and diamond, is one of the most promising diagnostic methods for high-energy, high repetition rate, laser-accelerated ions allowing a full on-line beam spectral characterization. A new analysis method for reconstructing the energy spectrum of high-energy laser-driven ion beams from TOF signals is hereby presented and discussed. The proposed method takes into account the detector's working principle, through the accurate calculation of the energy loss in the detector active layer, using Monte Carlo simulations. The analysis method was validated against well-established diagnostics, such as the Thomson parabola spectrometer, during an experimental campaign carried out at the Rutherford Appleton Laboratory (UK) with the high-energy laser-driven protons accelerated by the VULCAN Petawatt laser.
\end{abstract}

Published under license by AIP Publishing. https://doi.org/10.1063/1.5082746

\section{INTRODUCTION}

Over the last decades, the interest toward innovative particle acceleration techniques, alternative to conventional methods, has led to a growing effort in the study of high power laser-plasma interactions $s^{1,2}$ as sources of a varied range of particles. In particular, ultra-short multi-megaelectron volt laser-accelerated ions, if well controlled, could provide a promising alternative tool for dose delivery during radiobiological irradiations as well as, in a future perspective, for clinical treatments (hadrontherapy). ${ }^{3-8}$ In this framework, the development of novel instrumentation aimed at measuring with good accuracy ion beam characteristics, such as energy distribution, flux, and shot-to-shot reproducibility, represents a crucial step toward obtaining controlled beams, exploitable for applications. Well established detectors typically used in laser-driven acceleration experiments are, for instance, radiochromic films (RCFs), nuclear track detectors (CR-39), and image plates. 'They have been widely used for beam diagnosis with low-repetition rate laser systems operating in singleshot mode, and all require some form of processing after the 
exposure. Nevertheless, thanks to ongoing advances in laser technologies, the acceleration of high-energy ions at a high repetition rate is becoming possible and therefore the real-time diagnosis of laser-generated particles is a key point for the shot-to-shot monitoring of the beam parameters needed for applications. The Timeof-Flight (TOF) technique, so far used as on-line diagnostics for low-energy laser accelerated proton and ion beams, enables measurement of the ion maximum energy (cut-off), spectrum, and flux. $^{10,11}$

As discussed in the literature, ${ }^{11-17}$ detectors such as Faraday Cups (FC) and ion collectors (IC) and semiconductorlike detectors such as diamond and silicon carbide are typically placed at a finite distance from the target to measure TOF of accelerated particles. The TOF signal generated in such devices results from the contribution of the different ion species incoming with a specific TOF and, as a consequence, kinetic energy. According to the approach discussed in Refs. 18-21, TOF signals can be typically described as a convolution based on the so-called Maxwell-Boltzmann Shifted (MBS) functions defined for each ion species. The signal deconvolution by means of the MBS functions also allows investigating plasma parameters, such as the ion temperature and shift velocity, defined as the additional component to be considered due to the plasma center-of-mass velocity as it is reported in Ref. 19. When a single ion species, e.g. protons, is detected, the signal amplitude can be directly converted in an energy spectrum, considering the detector response to the incoming radiation. IC-FC and semiconductor detectors exhibit extremely different responses to charged particle radiation thus different approaches must be followed for the energy spectrum reconstruction from TOF signals. For instance, ion signal amplitudes generated in IC or FC devices depend on the collected charge, thus the absolute number of incoming particles can be obtained. On the other hand, for semiconductorlike detectors whose response depends on the energy deposited within the active detector layer and on the detector electron-hole pair energy creation, the signal amplitude needs to be converted. Nevertheless, the higher signal-to-noise ratio of semiconductorlike detectors compared to FC and IC detectors, coupled with their good performances, in terms of rise time and time resolution ${ }^{22,23}$ make silicon carbide and diamond detectors particularly suitable for the TOF diagnosis of high-energy particles.

A new analysis processing method is here proposed for reconstructing the ion energy spectrum from TOF signals generated by high-energy, laser-accelerated ions and acquired with semiconductorlike detectors. Such analysis procedure was successfully applied for the energy spectrum reconstruction of high-energy protons accelerated from the Vulcan PW laser system in a wide energy interval up to $30 \mathrm{MeV}$.

\section{A NEW PROCEDURE FOR PROTON ENERGY SPECTRUM RECONSTRUCTION}

As it is reported in Refs. 16, 17, and 21, a typical TOF signal displays the temporal evolution of the radiation and particles reaching the detector after being emitted from the laser-irradiated target: UV/X-rays promptly emitted when the laser hits the target surface generate the trigger signal in the detector, i.e., the so-called photopeak which is typically followed by a tail originated from fast electrons. Protons and heavier ion species, according to their energy and flight path, follow at longer TOF with a typical narrow peak (fast protons) and a broad signal (slow protons and ions). The kinetic energy of a given ion species, for instance, protons, is then calculated from the measured proton arrival time with respect to the photopeak $\left(t_{i o n}\right)$, knowing the flight path $\mathrm{L}$ and considering the time needed from UV and X-rays to travel the distance from the source to the detector $\left(t_{p h}\right)$

$$
T O F=t_{i o n}+t_{p h} \quad t_{p h}=\frac{L}{c},
$$

where $\mathrm{c}$ is the speed of light and TOF is the time of flight corrected for the photon flight path. The proton kinetic energy can be obtained by the well-known relativistic definition

$$
E_{k i n}=(\gamma-1) M_{p} c^{2} \quad \gamma=\frac{1}{\sqrt{\left(1-\beta^{2}\right)}} \quad \beta=\frac{L}{c T O F},
$$

where $M_{p}$ is the rest mass of proton or ion.

The absolute number of particles as well as the energy spectrum can be extracted from the signal amplitude, knowing the detector characteristics. As it is well known, the charge collected in semiconductorlike detectors due to $N$ incident particles, depositing energy $E$ in the active layer, can be expressed by

$$
Q=\frac{e N E}{\epsilon_{g}},
$$

where $\epsilon_{g}$ is the electron-hole pair energy creation, i.e., $7.78 \mathrm{eV}$ and $13 \mathrm{eV}$, respectively, for silicon carbide $(\mathrm{SiC})$ and diamond.

The detector can be directly connected to a fast oscilloscope for the acquisition with a typical time sampling of the order of $10^{-10} \mathrm{~s}$.

The current $i(t)$ measured with the oscilloscope corresponds to the detected charge $\delta \mathrm{Q}$ by a time interval $\delta \mathrm{t}$ and depends on the number of particles impinging the detector in the time interval $\delta \mathrm{t}$ as well as on their kinetic energy. Since both the number of particles and the kinetic energy of particles depend in turn on the time, i.e., the TOF, by performing the derivative in time of Eq. (3), it is possible to obtain the energy spectrum $d N / d E$ for a given ion species. The final expression for the energy spectrum is then obtained

$$
\frac{d N}{d E}=\frac{\epsilon_{g} i(t)}{e E^{2}}\left(-\frac{1}{2} t-\delta t\right)
$$

where $i(t)=V(t) / R$ with $\mathrm{V}(\mathrm{t})$ being generally the measured signal amplitude or the amplitude of the MBS function describing the original TOF signal and obtained from the signal deconvolution, $R$ being the total resistivity of the detector readout (including termination on the oscilloscope), $t$ corresponds to the measured TOF corrected with the light traveled distance, ${ }^{16} \delta \mathrm{t}$ is the oscilloscope time sampling (negligible by using fast scopes), and $L$ is the flight path. Integrating Eq. (4), the number of particles of a given species impinging on the detector can be extracted.

Considering the kinetic energy of the incident particles, two different regimes can occur as follows:

- Case 1. Particles having an incident kinetic energy such that they stop within the detector active thickness. In such case, the energy spectrum is reconstructed from Eq. (4) with the parameter $E$ corresponding to the kinetic energy obtained with Eq. (2). 
- Case 2. Particles having a sufficient kinetic energy to traverse the detector thickness, releasing only a fraction of their incident energy inside the active layer. In such case, the energy loss in the detector, corresponding to the incident energy measured with TOF technique, needs to be calculated.

So far proton energy spectra have been measured from TOF signals for low-energy protons when Case 1 occurred as it is reported in the literature. ${ }^{11,13,14}$ For Case 2, involving proton energies from few up to $100 \mathrm{MeV}$, a reliable reconstruction method is required to make the TOF technique an established diagnostic tool in view of its use in experiments with high-energy laser-driven ions. The approach proposed here takes into account the mechanism of signal formation in the specific detector employed in order to reconstruct the absolute number and energy spectrum of the impinging particles. Monte Carlo simulations are used to calculate the energy loss inside the detector active layer corresponding to the incident kinetic energy measured with the TOF technique.

The detector is simulated, using the Monte Carlo Geant $4^{24}$ code, in terms of material, density, and thickness, and a pointlike parallel beam is used as input source assuming a uniform energy distribution within the energy interval of interest. The particles generated in the Geant4-based application are tracked, and information, such as kinetic energy and position, is retrieved along the track. The energy deposited from each simulated particle within the detector thickness is then retrieved and can be related to the corresponding incident kinetic energy. In such way, a correlation between the kinetic energy and the energy loss can be obtained and used to extract from the experimental data the energy spectrum in the selected energy interval.

Such a method was used to analyze the TOF signals acquired in different laser-acceleration experiments using laser systems from few terawatt up to petawatt power accelerating protons with energies ranging from few $\mathrm{MeV}$ up to $30 \mathrm{MeV}$. $^{16,25,26}$ The procedure was validated against other well-established diagnostics and optimized adapting it according to the different experimental conditions and purposes.

In particular, the TOF technique employing diamond detectors was used to investigate proton acceleration during a recent experimental campaign carried out using the VULCAN PW laser at the Rutherford Appleton Laboratory (RAL). A laser pulse of wavelength $1.054 \mu \mathrm{m}$, duration $\sim 700 \mathrm{fs}$, and energy up to $\sim 400 \mathrm{~J}$ on target was focused onto a $25 \mu \mathrm{m}$-thick $\mathrm{Al}$ target leading to the acceleration (from surface contaminants) of protons and light ions, such as carbon and oxygen. A Thomson Parabola Spectrometer (TPS) coupled with image plates was placed in the backward direction at about $1.2 \mathrm{~m}$ from target, separating the ion species according to chargeto-mass ratio and providing the ion energy cut-off and spectra measurements using the analysis method and the calibration reported in Refs. 27 and 28. A $100 \mu \mathrm{m}$ thick polychrystalline diamond detector was placed at the target front side at about $2.35 \mathrm{~m}$ (P1) from the target location, with an applied voltage of $200 \mathrm{~V}$. The experimental setup is shown in Fig. 1.

As one can see in Fig. 2, the acquired TOF signal is composed of a small photopeak (7-10 ns) generated by plasma soft X-ray/ultraviolet (XUV) emission and a broad peak (32-140 ns) resulted as the sum of protons and other contaminants. According to TPS measurements, carbon and oxygen ions in different charge

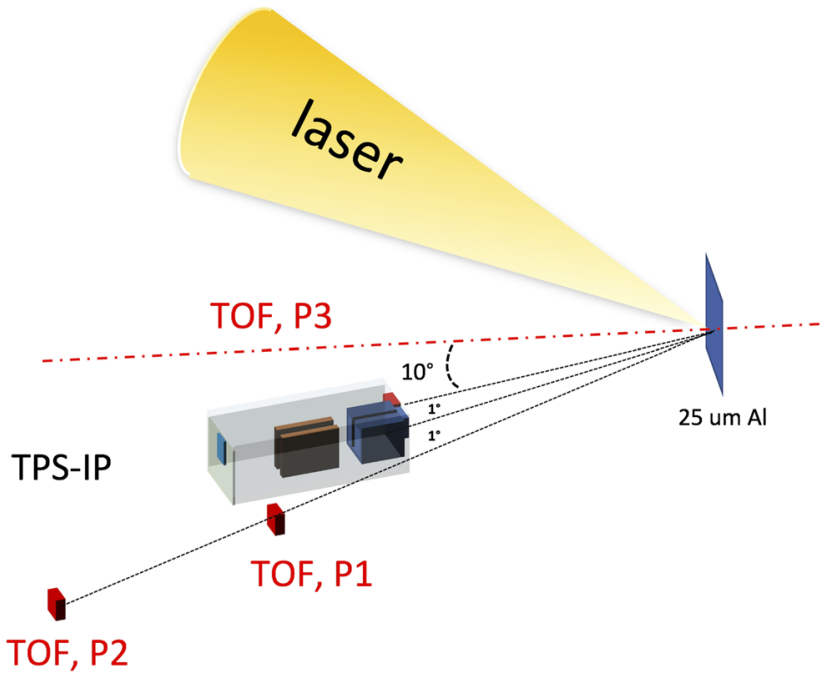

FIG. 1. Experimental setup: the laser incidence angle (laser-target normal) was about $20^{\circ}$ and the TPS-IP was placed at about $10^{\circ}$. The three positions of the diamond detector used for TOF measurements (TOF) are also indicated: P1 and P2 have, respectively, a $2.35 \mathrm{~m}$ and $4.10 \mathrm{~m}$ flight path. P3 indicates the position of the diamond detector placed alongside the TPS pinhole at about $1.2 \mathrm{~m}$ from the target.

states are accelerated together with protons, with a maximum energy per nucleon not exceeding $4 \mathrm{AMeV}$, which for carbon ions corresponds to a TOF at 2.35 of about $80 \mathrm{~ns}$. As a consequence, the TOF signal in the time interval $32-80 \mathrm{~ns}$ is uniquely originated from protons and the energy distribution can be reconstructed following the developed procedure.

The LISE++ analytical code was used to calculate the minimum proton energy needed to traverse the detector thickness. Such calculation, resulting of about $5 \mathrm{MeV}$, allowed us to distinguish between case $1\left(\mathrm{E}_{k i n}<5 \mathrm{MeV}\right)$ and case $2\left(\mathrm{E}_{k i n}>5 \mathrm{MeV}\right)$. A Monte Carlo simulation (Geant4) reproducing the experimental condition, namely, protons with energies higher than $5 \mathrm{MeV}$ impinging on a $100 \mu \mathrm{m}$ thick diamond detector, was then performed to retrieve the

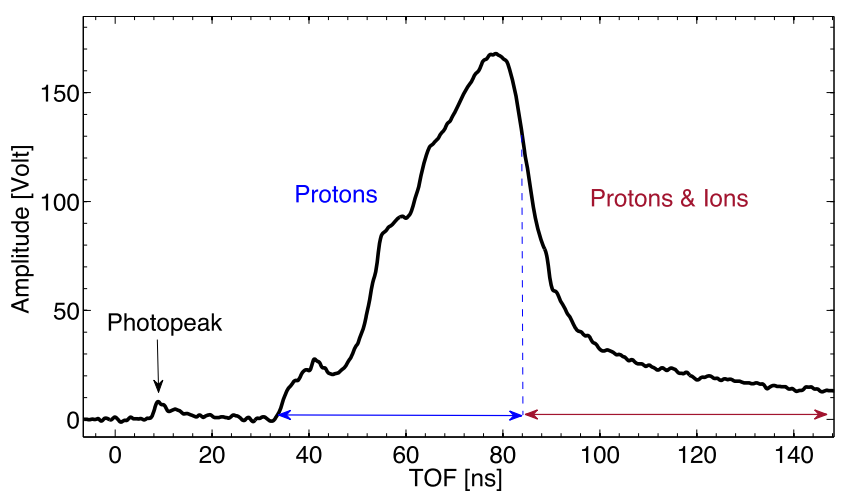

FIG. 2. TOF signal acquired with a $100 \mu \mathrm{m}$ thick polychristallyne diamond detector during the experiment carried out with Vulcan Petawatt. 


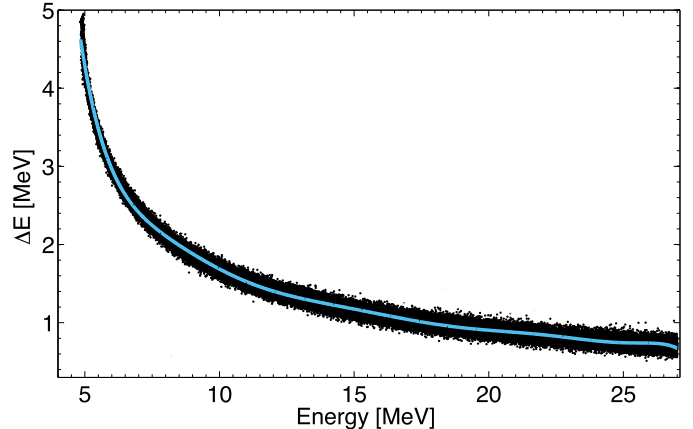

FIG. 3. Energy loss $(\Delta E)$ simulated with Geant4 for protons with energies ranging from 5 to $27 \mathrm{MeV}$ in $100 \mu \mathrm{m}$-thick diamond.

corresponding energy loss and reconstruct the energy spectrum for case 2. The energy loss $(\Delta \mathrm{E})$ as a function of the incident kinetic energy is thus obtained by means of the Geant 4 simulations as it is shown in Fig. 3.

As it is expected, the energy loss calculation takes into account the energy straggling effect, originating from stochastic fluctuations in the energy loss of protons. The FWHM of such energy loss distribution, typically of the order of hundreds of kilo-electron-volt, was considered in the analysis as a contribution to the uncertainty on the energy loss calculation.

A function relating the kinetic energy and the average energy loss was obtained through a polynomial fit (light blue line in Fig. 3). In such a case, a 9th order polynomial function $\left(\sum_{n=0}^{9} p_{n} E_{k i n}^{n}\right)$ has been used to fit the data in Fig. 3. The parameters resulting from the fit were then used to associate an energy loss to the kinetic energies calculated from the measured TOF values.

The energy spectrum obtained in the range 5-27 MeV is shown in Fig. 4. Integrating the energy distribution, a number of protons per solid angle of $(5 \pm 1) \times 10^{12}$ protons/sr were estimated. The uncertainty on the number of proton per steradian is calculated applying the error propagation on Eq. (4) and considering the statistical uncertainty. An uncertainty on the energy loss calculation is

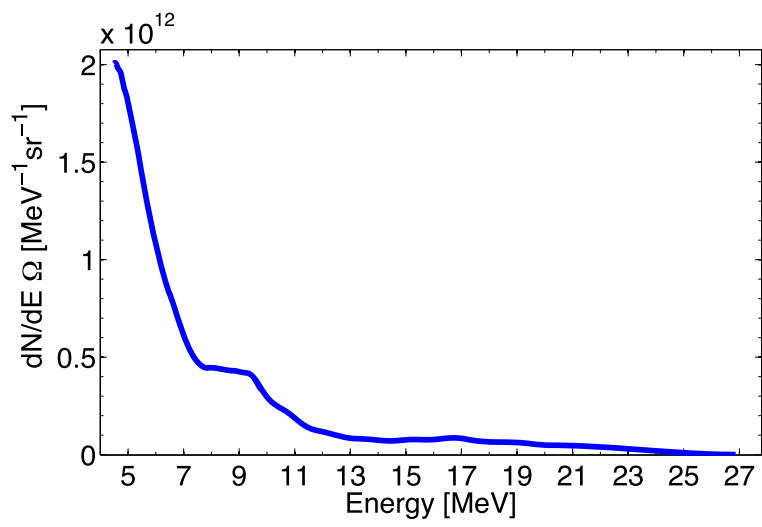

FIG. 4. Proton energy spectrum reconstructed following the proposed procedure from the TOF signal shown in Fig. 2. of the order of $10 \%$ and was evaluated taking into account the energy straggling effect. Uncertainty on the solid angle of the order of $0.1 \%$ was considered.

As it is shown in Fig. 2, besides protons, other ion species can be accelerated from the target and will overlap in the detector signal with protons having the same TOF.

In Fig. 2, this is the case for TOF $>80 \mathrm{~ns}$, where the TOF signal results from the overlap of different ions (carbon, oxygen, and protons), and the single species contribution as well as the corresponding energy distributions cannot be discriminated.

Metallic or plastic absorbers with different thicknesses are typically placed in front of the detector to select the high-energy proton component and filter the ions and the high-flux low-energy protons, which could saturate the detector.

With an appropriate filter, a TOF signal uniquely due to protons can be obtained and the proton energy spectrum can be then extracted. Figure 5 shows a TOF signal acquired with a $100 \mu \mathrm{m}$ thick diamond detector placed at about $4 \mathrm{~m}$ (P2) from the target in the backward direction (Fig. 1). A $50 \mu \mathrm{m} \mathrm{Al}$ foil absorber was used in front of the detector. According to the calculations performed with the code LISE++, such absorber stops protons, carbon, and oxygen ions with energies up to $2.23 \mathrm{MeV}, 3.7 \mathrm{AMeV}$, and 4.2 $\mathrm{AMeV}$, respectively.

The TOF values corresponding to such energies at $4.10 \mathrm{~m}$ for protons, carbon, and oxygen ions are, respectively, $200 \mathrm{~ns}, 153 \mathrm{~ns}$, and 142 ns. Coupling together these considerations and the maximum ion energies measured with the TPS of about $4 \mathrm{MeV} / \mathrm{n}$, which corresponds to a TOF of $148 \mathrm{~ns}$ at $4.10 \mathrm{~m}$, three time intervals can be identified in the TOF signal shown in Fig. 5: (1) 65-148 ns, the signal can be attributed uniquely to higher-energy proton contribution; (2) 148-153 ns, the signal results from the overlap of $\mathrm{H}^{+}, \mathrm{C}^{12}$, and $\mathrm{O}^{16}$ ions in different charge states; and (3) 153-200 ns, the signal originates only from low-energy proton component (from $2.23 \mathrm{MeV}$ up to $3.7 \mathrm{MeV}$ ).

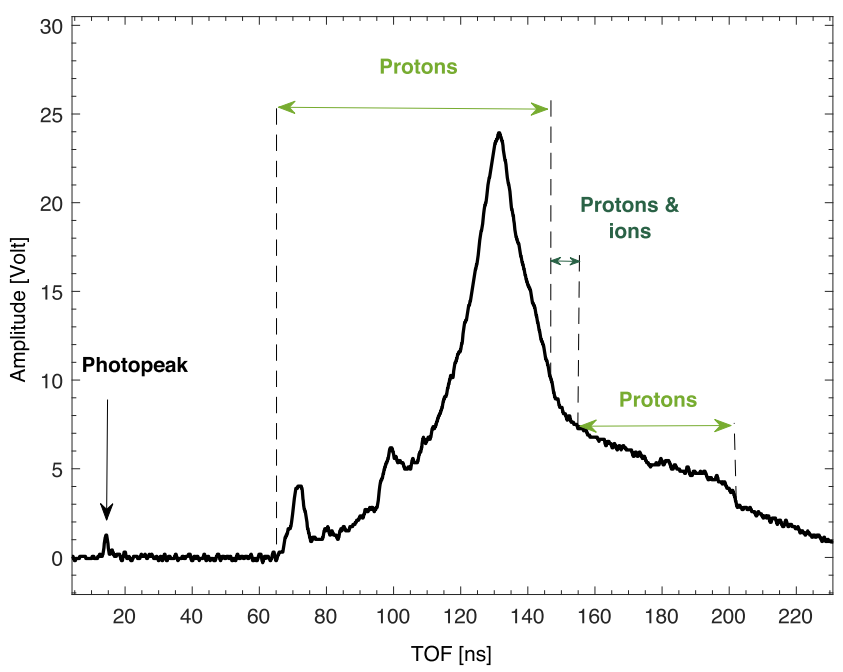

FIG. 5. TOF signal acquired in the backward direction with the $100 \mu \mathrm{m}$ thick diamond detector for flight path $=4.10 \mathrm{~m}$. A $50 \mu \mathrm{m} \mathrm{Al}$ foil was used to stop heavy ions. Proton and ion contributions in the three different time intervals are shown. 


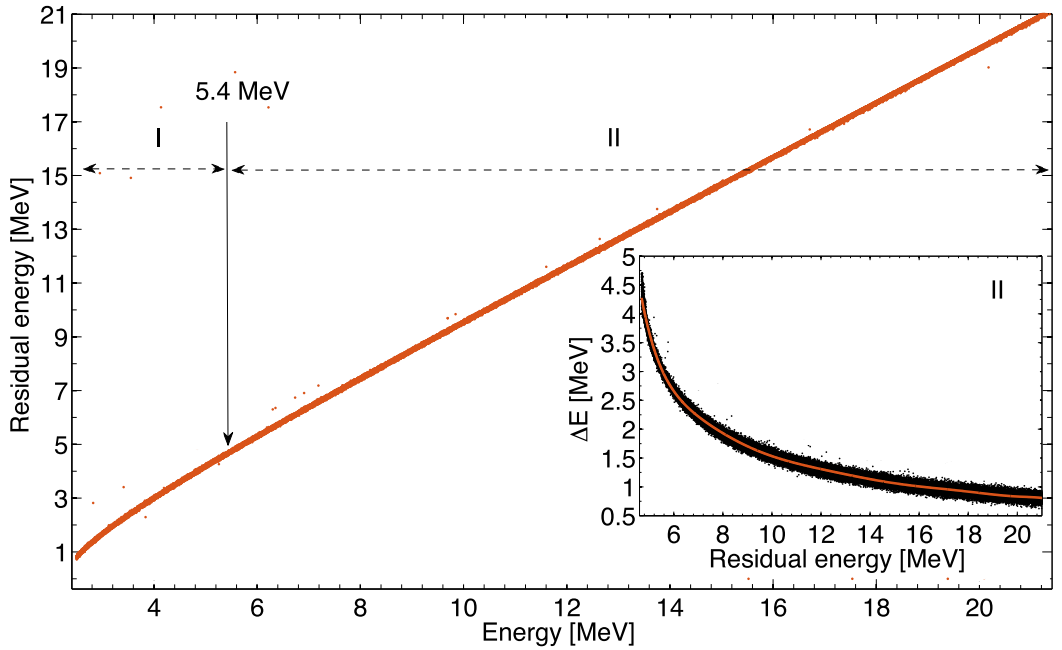

FIG. 6. Residual energy as a function of incident kinetic energy in the energy interval between $2.2 \mathrm{MeV}$ and $21 \mathrm{MeV}$ estimated with MC simulation. Inset: Energy loss within the detector thickness as a function of the residual energy (case II) obtained with the Geant4 simulation.
In region $(2), \mathrm{H}^{+}, \mathrm{C}^{12}$, and $\mathrm{O}^{16}$ contributions cannot be disentangled from the TOF signal since the signal is a convolution of different ion species and charge states. The proton energy distribution was therefore extracted in the regions (1) and (3) following the approach described earlier. In the presence of absorbers, Monte Carlo simulations are also needed to estimate, for particles traversing the absorber, the fraction of the incident energy lost within the absorber thickness and the residual energy in the detector thickness. Two cases can occur:

I. Particles with residual energy after the absorber insufficient to traverse the detector thickness. These particles stop within the detector active layer and the variable $E$ in Eq. (4) correspond to the particle residual energy.

II. Particles with residual energy after the absorber sufficient to traverse the detector thickness. Considering the residual energy after the absorber, the corresponding energy released within the detector thickness has to be calculated with the help of Monte Carlo simulations.

Figure 6 shows the residual energy of protons traversing a $50 \mu \mathrm{m} \mathrm{Al}$ foil simulated with Geant 4 as a function of the incident proton energy in the energy range from $2.2 \mathrm{MeV}$ up to $21 \mathrm{MeV}$ for the shot shown in Fig. 5. The two regions I and II are indicated in Fig. 6. The proton energy corresponding to the transition between the two cases, i.e., $5.4 \mathrm{MeV}$, is also shown. Two different simulations have been performed for the two regions, according to the value calculated with the LISE++ code: for protons with an incident energy ranging from $2.2 \mathrm{MeV}$ up to $5.4 \mathrm{MeV}$ (region I), the residual energy after the filter is calculated by means of simulations and can be directly used for the energy spectrum reconstruction; on the other hand, for protons in region II, the energy loss within the detector thickness as a function of the residual energy has to be calculated (inset in Fig. 6) similarly to the case shown in Fig. 3. The energy distribution corresponding to the TOF signal in Fig. 5 is shown in Fig. 7: the gap between $3.7 \mathrm{MeV}$ and $4 \mathrm{MeV}$ corresponds to the region (2) in Fig. 5, where carbon ions contribute to the TOF signal besides protons.
The new analysis method presented in this work was also used to analyze TOF data acquired with the diamond detector placed alongside the TPS (backward direction) at the same distance $(1.22 \mathrm{~m})$ and at about the same angle $\left(\sim 1^{\circ}\right)$ for several shots as it is shown in Fig. 1. This allowed validating the TOF procedure through a direct comparison between the two diagnostics. Figure 8 shows the number of protons per solid angle ( $\mathrm{Np} /$ steradian) obtained with the TOF developed procedure and with the TPS in the energy interval ranging between $13 \mathrm{MeV}$ and $18 \mathrm{MeV}$ for 6 consecutive shots. The energy range corresponds to the energy region common to all the shots analyzed. A similar comparison between the maximum proton energies measured with the two diagnostics has been also performed for the same shots as it is shown in Fig. 9. The TPS uncertainty was estimated to be about $15 \%$, considering the statistical and the calibration uncertainties. The results in Figs. 8 and 9 confirm the good agreement between the two diagnostics, both sensitive to shot to shot fluctuations, indicating the good reliability of the

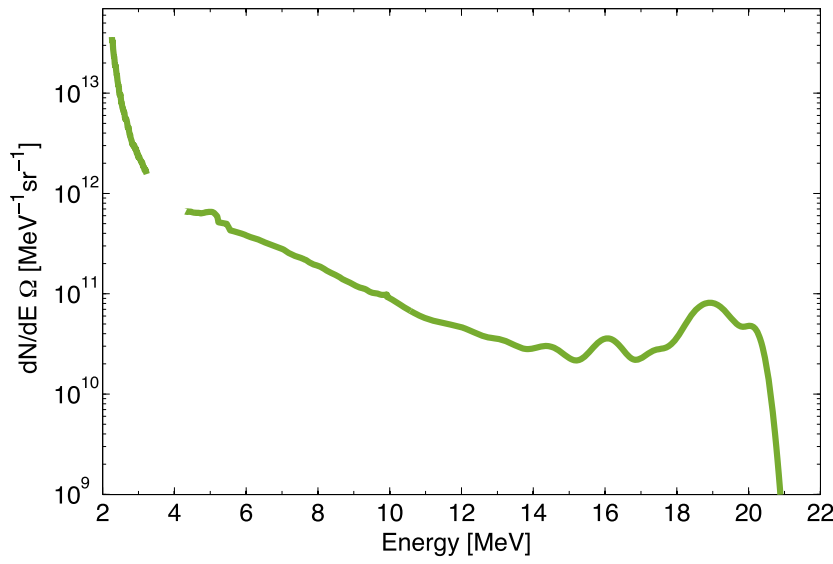

FIG. 7. TOF signal acquired in the backward direction with the $100 \mu \mathrm{m}$ thick diamond detector, flight path $=4.10 \mathrm{~m}$. A $50 \mu \mathrm{m}$ Al foil was used to stop heavy ions. Proton and ion contributions in 3 different time intervals are shown. 


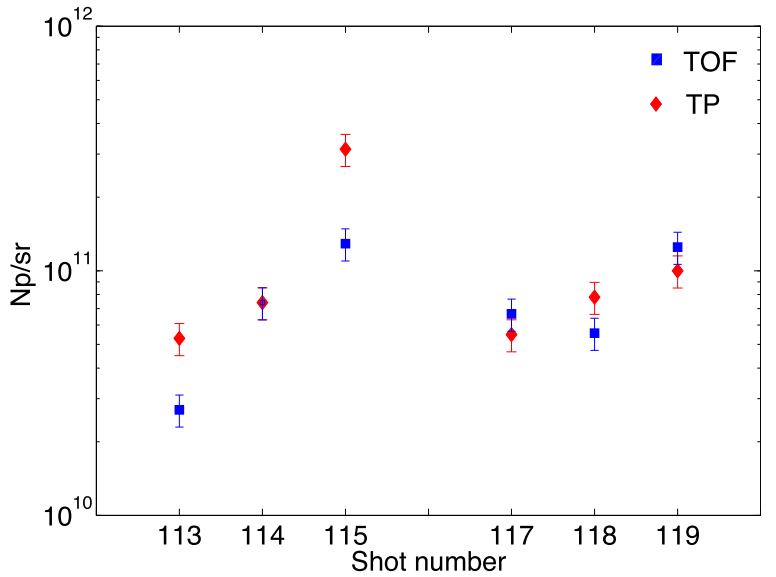

FIG. 8. Number of protons per solid angle in the energy range 13-18 MeV reconstructed with TOF technique (blue solid squares) and TPS images (red solid diamonds) for 6 consecutive shots.

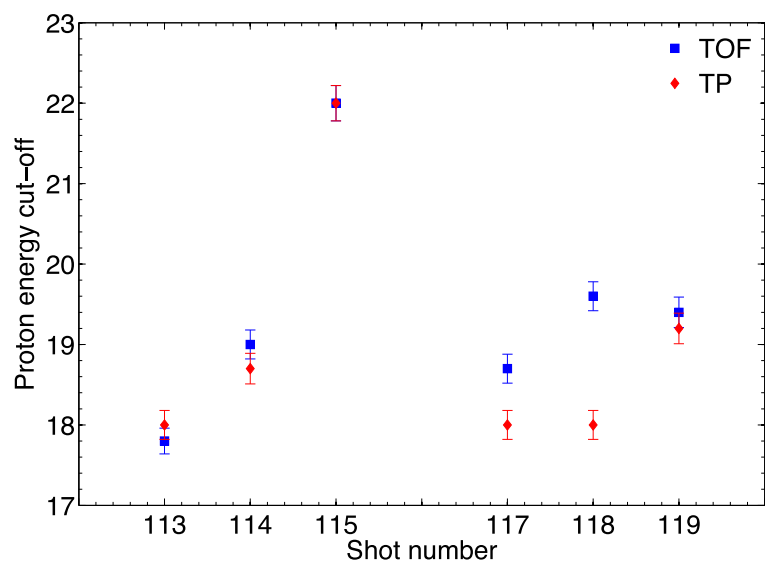

FIG. 9. Proton energy cutoffs measured with the TOF technique (blue solid squares) and TPS images (red solid diamonds) for 6 consecutive shots.

developed TOF analysis method and its potential in the reconstruction of high-energy ion spectra with a good accuracy.

\section{CONCLUSIONS}

An analysis procedure, which allows converting the TOF signals measured with diamond detectors in energy distribution for a given ion species, is proposed here. It takes into account the mechanism for signal formation in semiconductorlike detectors, required to extract the energy spectrum. The method hereby described was validated during an experimental campaign carried out at the Vulcan PW laser facility (RAL), and the obtained energy spectra are here shown together with a direct comparison with the measurement performed with a TPS, taken as a reference diagnostics during the experiment. The good agreement between the two diagnostics, in terms of energy cutoffs and number of protons/steradian, confirms the reliability of the analysis procedure. The experiment shows that when different ion species are accelerated, the TOF technique requires complementary information from the TPS, i.e., ion species and their maximum energies, to disentangle protons from the ion contribution in the TOF signal. New approaches based on the use of a stand-alone multistage diamond detector are currently under study allowing to solve the necessity to obtain the measurements for the ions from an external detector and making TOF technique self-consistent. On the other hand, such TOF detectors offer high prospects for the diagnosis of high-repetition rate (up to $10 \mathrm{~Hz}$ ) accelerated protons and, with a suitable, automated procedure, could provide information on the shot-to-shot energy distribution and flux in real-time. In this perspective, a database containing the results of simulations for a given detector type and energy intervals would be a key requirement for a fast, automated signal analysis. Data associated with the research published in this article can be accessed at https://doi.org/10.17034/037cc5d3-409a-47f2ba2c-f4b56f1a9818.

\section{ACKNOWLEDGMENTS}

This work has been supported by the V committee of INFN (Italian Institute for Nuclear Physics) within the ELIMED activities, by the MIUR and by the project Advanced research using high intensity laser produced photons and particles (Grant No. CZ.02.1.01/0.0/0.0/16_019/0000789) from the European Regional Development Fund (ADONIS). The results of the Project No. LQ1606 were also obtained with the financial support of the Ministry of Education, Youth and Sports as part of targeted support from the National Programme of Sustainability II. Moreover, the authors acknowledge the facility access provided by the Science and Technology Facility Council and the support from the laser and technical staff and target fabrication group at the Rutherford Appleton Laboratory.

\section{REFERENCES}

${ }^{1}$ A. Macchi, S. Veghini, T. V. Liseykina, and F. Pegoraro, "Radiation pressure acceleration of ultrathin foils," New J. Phys. 12(4), 045013 (2010).

${ }^{2}$ A. Macchi, M. Borghesi, and M. Passoni, "Ion acceleration by superintense laser-plasma interaction,” Rev. Mod. Phys. 85(2), 751-793 (2013); e-print arXiv:1302.1775.

${ }^{3}$ S. V. Bulanov, "Laser ion acceleration for hadron therapy," in Proceedings-2014 International Conference Laser Optics, LO 2014 [Phys.-Usp. 57(12), 1149-1179 (2014)].

${ }^{4}$ S. Busold, A. Almomani, V. Bagnoud, W. Barth, S. Bedacht, A. Blažević, O. Boine-Frankenheim, C. Brabetz, T. Burris-Mog, T. Cowan, O. Deppert, M. Droba, H. Eickhoff, U. Eisenbarth, K. Harres, G. Hoffmeister, I. Hofmann, O. Jaeckel, R. Jaeger, M. Joost, S. Kraft, F. Kroll, M. Kaluza, O. Kester, Z. Lecz, T. Merz, F. Nürnberg, H. Al-Omari, A. Orzhekhovskaya, G. Paulus, J. Polz, U. Ratzinger, M. Roth, G. Schaumann, P. Schmidt, U. Schramm, G. Schreiber, D. Schumacher, T. Stoehlker, A. Tauschwitz, W. Vinzenz, F. Wagner, S. Yaramyshev, and B. Zielbauer, "Shaping laser accelerated ions for future applications-The LIGHT collaboration," Nucl. Instrum. Methods Phys. Res., Sect. A 740, 94-98 (2014).

${ }^{5}$ G. Cirrone, G. Cuttone, F. Romano, F. Schillaci, V. Scuderi, A. Amato, G. Candiano, M. Costa, G. Gallo, G. Larosa, G. Korn, R. Leanza, R. Manna, M. Maggiore, V. Marchese, D. Margarone, G. Milluzzo, G. Petringa, and A. Tramontana, "Design and status of the ELIMED beam line for laser-driven ion beams,” Appl. Sci. 5(3), 427-445 (2015). 
${ }^{6}$ U. Masood, T. E. Cowan, W. Enghardt, K. M. Hofmann, L. Karsch, F. Kroll, U. Schramm, J. J. Wilkens, and J. Pawelke, "A light-weight compact proton gantry design with a novel dose delivery system for broad-energetic laser-accelerated beams," Phys. Med. Biol. 62(13), 5531-5555 (2017).

${ }^{7}$ D. Margarone, G. Cirrone, G. Cuttone, A. Amico, L. Andò, M. Borghesi, S. Bulanov, S. Bulanov, D. Chatain, A. Fajstavr, L. Giuffrida, F. Grepl, S. Kar, J. Krasa, D. Kramer, G. Larosa, R. Leanza, T. Levato, M. Maggiore, L. Manti, G. Milluzzo, B. Odlozilik, V. Olsovcova, J.-P. Perin, J. Pipek, J. Psikal, G. Petringa, J. Ridky, F. Romano, B. Rus, A. Russo, F. Schillaci, V. Scuderi, A. Velyhan, R. Versaci, T. Wiste, M. Zakova, and G. Korn, "ELIMAIA: A laser-driven ion accelerator for multidisciplinary applications," Quantum Beam Sci. 2(2), 8 (2018). ${ }^{8}$ A. Higginson, R. J. Gray, M. King, R. J. Dance, S. D. R. Williamson, N. M. H. Butler, R. Wilson, R. Capdessus, C. Armstrong, J. S. Green, S. J. Hawkes, P. Martin, W. Q. Wei, S. R. Mirfayzi, X. H. Yuan, S. Kar, M. Borghesi, R. J. Clarke, D. Neely, and P. McKenna, "Near-100 MeV protons via a laser-driven transparency-enhanced hybrid acceleration scheme," Nat. Commun. 9(1), 724 (2018).

${ }^{9}$ P. R. Bolton, M. Borghesi, C. Brenner, D. C. Carroll, C. De Martinis, A. Flacco, V. Floquet, J. Fuchs, P. Gallegos, D. Giove, J. S. Green, S. Green, B. Jones, D. Kirby, P. McKenna, D. Neely, F. Nuesslin, R. Prasad, S. Reinhardt, M. Roth, U. Schramm, G. G. Scott, S. Ter-Avetisyan, M. Tolley, G. Turchetti, and J. J. Wilkens, "Instrumentation for diagnostics and control of laser-accelerated proton (ion) beams," Phys. Med. 30(3), 255-270 (2014).

${ }^{10}$ D. Margarone, L. Torrisi, S. Cavallaro, E. Milani, G. Verona-Rinati, M. Marinelli, C. Tuvé, L. Láska, J. Krasa, M. Pfeifer, E. Krousky, J. Ullshmied, L. Ryc, A. Mangione, and A. M. Mezzasalma, "Diamond detectors for characterization of laser-generated plasma," Radiat. Eff. Defects Solids 163(4-6), 463-470 (2008).

${ }^{11}$ D. Margarone, J. Krsa, L. Giuffrida, A. Picciotto, L. Torrisi, T. Nowak, P. Musumeci, A. Velyhan, J. Prokpek, L. Lska, T. Mocek, J. Ullschmied, and B. Rus, "Full characterization of laser-accelerated ion beams using Faraday cup, silicon carbide, and single-crystal diamond detectors," J. Appl. Phys. 109(10), 103302 (2011).

${ }^{12}$ D. Margarone, J. Krasa, J. Prokupek, A. Velyhan, L. Torrisi, A. Picciotto, L. Giuffrida, S. Gammino, P. Cirrone, M. Cutroneo, F. Romano, E. Serra, A. Mangione, M. Rosinski, P. Parys, L. Ryc, J. Limpouch, L. Laska, K. Jungwirth, J. Ullschmied, T. Mocek, G. Korn, and B. Rus, "New methods for high current fast ion beam production by laser-driven acceleration," Rev. Sci. Instrum. 83(2), 02B307 (2012).

${ }^{13}$ G. Bertuccio, D. Puglisi, L. Torrisi, and C. Lanzieri, "Silicon carbide detector for laser-generated plasma radiation,” Appl. Surf. Sci. 272, 128-131 (2013).

${ }^{14} \mathrm{M}$. Marinelli, E. Milani, G. Prestopino, C. Verona, G. Verona-Rinati, M. Cutroneo, L. Torrisi, D. Margarone, A. Velyhan, J. Krasa, and E. Krousky, "Analysis of laser-generated plasma ionizing radiation by synthetic single crystal diamond detectors," Appl. Surf. Sci. 272, 104-108 (2013).

${ }^{15} \mathrm{P}$. Musumeci, M. Cutroneo, L. Torrisi, A. Velyhan, M. Zimbone, and L. Calcagno, "Silicon carbide detectors for diagnostics of ion emission from laser plasmas,” Phys. Scr. T161, 014021 (2014).

${ }^{16}$ G. Milluzzo, V. Scuderi, A. G. Amico, M. Borghesi, G. A. Cirrone, G. Cuttone, M. D. Napoli, D. Doria, J. Dostal, G. Larosa, R. Leanza, D. Margarone, G. Petringa, J. Pipek, L. Romagnani, F. Romano, F. Schillaci, and A. Velyhan, "Laseraccelerated ion beam diagnostics with TOF detectors for the ELIMED beam line," J. Instrum. 12(2), C02025 (2017)

${ }^{17}$ V. Scuderi, G. Milluzzo, A. Alejo, A. Amico, N. Booth, G. Cirrone, D. Doria, J. Green, S. Kar, G. Larosa, R. Leanza, D. Margarone, P. McKenna, H. Padda, G. Petringa, J. Pipek, L. Romagnani, F. Romano, F. Schillaci, M. Borghesi, G. Cuttone, and G. Korn, "Time of flight based diagnostics for high energy laser driven ion beams," J. Instrum. 12(03), C03086 (2017).
${ }^{18}$ A. Miotello and R. Kelly, "On the origin of the different velocity peaks of particles sputtered from surfaces by laser pulses or charged-particle beams," Appl. Surf. Sci. 138-139, 44-51 (1999).

${ }^{19} \mathrm{R}$. Kelly and R. Dreyfus, "On the effect of Knudsen-layer formation on studies of vaporization, sputtering, and desorption," Surf. Sci. 198(1-2), 263-276 (1988).

${ }^{20} \mathrm{~J}$. Krása, "Gaussian energy distribution of fast ions emitted by laser-produced plasmas,” Appl. Surf. Sci. 272, 46-49 (2013).

${ }^{21}$ G. Milluzzo, V. Scuderi, A. G. Amico, G. A. Cirrone, G. Cuttone, M. De Napoli, J. Dostal, G. Larosa, R. Leanza, D. Margarone, G. Petringa, J. Pipek, F. Romano, F. Schillaci, and A. Velyhan, "TOF technique for laser-driven proton beam diagnostics for the ELIMED beamline," J. Instrum. 12(3), C03044 (2017).

${ }^{22}$ M. De Napoli, F. Giacoppo, G. Raciti, E. Rapisarda, and C. Sfienti, "Light ions response of $4 \mathrm{H}-\mathrm{SiC}$ Schottky diodes with different dopant concentration," Nucl. Phys. B, Proc. Suppl. 197(1), 198-201 (2009).

${ }^{23}$ N. Randazzo, S. Aiello, G. Chiodini, G. A. Cirrone, G. Cuttone, M. De Napoli, V. Giordano, S. Kwan, E. Leonora, F. Longhitano, D. Lo Presti, L. Moroni, C. Pugliatti, R. Rivera, V. Scuderi, V. Sipala, C. Stancampiano, C. Tuve, and L. Uplegger, "Comparative timing performances of S-CVD diamond detectors with different particle beams and readout electronics," in 2012 IEEE Nuclear Science Symposium and Medical Imaging Conference Record (NSS/MIC) (IEEE, 2012), pp. 1949-1951.

${ }^{24}$ J. Allison, K. Amako, J. Apostolakis, H. Araujo, P. A. Dubois, M. Asai, G. Barrand, R. Capra, S. Chauvie, R. Chytracek, G. A. Cirrone, G. Cooperman, G. Cosmo, G. Cuttone, G. G. Daquino, M. Donszelmann, M. Dressel, G. Folger, F. Foppiano, J. Generowicz, V. Grichine, S. Guatelli, P. Gumplinger, A. Heikkinen, I. Hrivnacova, A. Howard, S. Incerti, V. Ivanchenko, T. Johnson, F. Jones, T. Koi, R. Kokoulin, M. Kossov, H. Kurashige, V. Lara, S. Larsson, F. Lei, F. Longo, M. Maire, A. Mantero, B. Mascialino, I. McLaren, P. M. Lorenzo, K. Minamimoto, K. Murakami, P. Nieminen, L. Pandola, S. Parlati, L. Peralta, J. Perl, A. Pfeiffer, M. G. Pia, A. Ribon, P. Rodrigues, G. Russo, S. Sadilov, G. Santin, T. Sasaki, D. Smith, N. Starkov, S. Tanaka, E. Tcherniaev, B. Tomé, A. Trindade, P. Truscott, L. Urban, M. Verderi, A. Walkden, J. P. Wellisch, D. C. Williams, D. Wright, H. Yoshida, and M. Peirgentili, "Geant4 developments and applications," IEEE Trans. Nucl. Sci. 53(1), 270-278 (2006).

${ }^{25}$ L. Giuffrida, K. Svensson, J. Psikal, M. Dalui, H. Ekerfelt, I. G. Gonzalez, O. Lundh, A. Persson, P. Lutoslawski, V. Scuderi, J. Kaufman, T. Wiste, T. Lastovicka, A. Picciotto, A. Bagolini, M. Crivellari, P. Bellutti, G. Milluzzo, G. A. P. Cirrone, J. Magnusson, A. Gonoskov, G. Korn, C.-G. Wahlström, and D. Margarone, "Manipulation of laser-accelerated proton beam profiles by nanostructured and microstructured targets," Phys. Rev. Accel. Beams 20, 081301 (2017).

${ }^{26}$ D. Margarone, A. Velyhan, J. Dostal, J. Ullschmied, J. P. Perin, D. Chatain, S. Garcia, P. Bonnay, T. Pisarczyk, R. Dudzak, M. Rosinski, J. Krasa, L. Giuffrida, J. Prokupek, V. Scuderi, J. Psikal, M. Kucharik, M. De Marco, J. Cikhardt, E. Krousky, Z. Kalinowska, T. Chodukowski, G. A. Cirrone, and G. Korn, "Proton acceleration driven by a nanosecond laser from a cryogenic thin solid-hydrogen ribbon," Phys. Rev. X 6(4), 041030 (2016).

${ }^{27}$ A. Alejo, S. Kar, A. Tebartz, H. Ahmed, S. Astbury, D. C. Carroll, J. Ding, D. Doria, A. Higginson, P. McKenna, N. Neumann, G. G. Scott, F. Wagner, M. Roth, and M. Borghesi, "High resolution Thomson parabola spectrometer for full spectral capture of multi-species ion beams," Rev. Sci. Instrum. 87(8), 083304 (2016).

${ }^{28}$ D. Doria, S. Kar, H. Ahmed, A. Alejo, J. Fernandez, M. Cerchez, R. J. Gray, F. Hanton, D. A. MacLellan, P. McKenna, Z. Najmudin, D. Neely, L. Romagnani, J. A. Ruiz, G. Sarri, C. Scullion, M. Streeter, M. Swantusch, O. Willi, M. Zepf, and M. Borghesi, "Calibration of BAS-TR image plate response to high energy (3-300 $\mathrm{MeV}$ ) carbon ions,” Rev. Sci. Instrum. 86(12), 123302 (2015). 